\title{
Does metformin improve vascular health in children with type 1 diabetes? Protocol for a one year, double blind, randomised, placebo controlled trial
}

\author{
Jemma Anderson ${ }^{1,2^{*}}$, Alexia S Peña ${ }^{1,2}$, Thomas Sullivan ${ }^{3}$, Roger Gent ${ }^{4}$, Bronwen D'Arcy ${ }^{5}$, Timothy Olds ${ }^{6}$, \\ Brian Coppin ${ }^{7}$ and Jennifer Couper ${ }^{1,2}$
}

\begin{abstract}
Background: Cardiovascular disease is the leading cause of mortality in Type 1 diabetes (T1D). Vascular dysfunction is an early and critical event in the development of cardiovascular disease. Children with T1D have vascular dysfunction therefore early interventions to improve vascular health are essential to reduce cardiovascular mortality in T1D. Metformin is an insulin sensitising agent which is known to improve vascular health outcomes in type 2 diabetes (T2D) and other individuals with insulin resistance. It has been used safely in children and adolescents with T2D for over 10 years. This study aims to assess the effect of metformin on vascular health in children with T1D.

Methods/Design: This study is a 12 month, double blind, randomised, placebo controlled trial to determine the effect of metformin on vascular health in children (age 8-18) with T1D. The sample size is 76 with 38 children in the metformin group and 38 children in the placebo group. Vascular health and biochemical markers will be measured at baseline, 3, 6 and 12 months. Vascular function will be measured using flow mediated dilatation and glyceryl trinitrate mediated dilatation of the brachial artery and vascular structure will be measured with carotid and aortic intima media thickness, using standardised protocols.
\end{abstract}

Discussion: This study will be the first to investigate the effect of metformin on vascular health in children with T1D. It will provide important information on a potential intervention to improve cardiovascular morbidity and mortality in this population at high risk from cardiovascular disease.

Trial registration: Australia New Zealand Clinical Trials Registry ACTRN12611000148976

Keywords: Endothelial function, Metformin, Type 1 diabetes, Child

\section{Background}

Vascular dysfunction and cardiovascular disease in Type 1 diabetes

XCardiovascular disease remains the leading cause of mortality in Type 1 Diabetes (T1D), despite significant developments in management over the past 20 years [1,2]. Children with T1D have evidence of cardiovascular abnormalities such as vascular endothelial and smooth muscle dysfunction and increased intima media thickness [3-5]. Vascular dysfunction precedes clinically evident

\footnotetext{
* Correspondence: jemma.anderson@adelaide.edu.au

'School of Paediatrics and Reproductive Health, University of Adelaide,

Adelaide, Australia

2Department of Paediatric Endocrinology, Women's and Children's Hospital,

Adelaide, Australia

Full list of author information is available at the end of the article
}

vascular disease [6] and is potentially reversible. Although vascular complications are rarely seen during childhood, their pathogenesis begins soon after diagnosis and accelerates during puberty [7]. Vascular dysfunction in T1D is also accelerated by poor glycaemic control, overweight and obesity, genetic predisposition and insulin resistance. Early interventions to improve cardiovascular health in T1D are essential to reduce the burden of cardiovascular morbidity and mortality.

The doubling in incidence of T1D in childhood in Australia over the last 20 years parallels the overweight/ obesity epidemic in Western childhood populations. In addition, age of diagnosis of T1D has decreased over the last 20 years and younger age of T1D onset is associated in some populations with higher Body Mass Index (BMI)

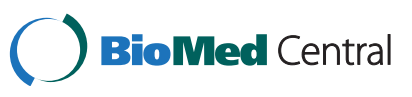


at diagnosis $[8,9]$. The T1D population is susceptible to the population shift in BMI, with $65 \%$ of adults in a US cohort with T1D in the overweight or obese BMI range [10]. Higher BMI is associated with higher intima media thickness (IMT) blood pressure and dyslipidaemia [11].

The prevalence of overweight and obesity in children with T1D is higher than the normal population, at just over $38.5 \%$ in a Dutch cohort [12], 22.1\% in a US cohort (compared with $16.1 \%$ in the normal population) [13] and $31 \%$ in our local South Australian cohort [14]. This is associated with other cardiovascular risk factors including hypertension, dyslipidaemia, and metabolic syndrome. Overweight and obesity may have additional clinical consequences with the associated higher insulin resistance contributing to vascular disease [12,15].

Adolescence is a critical time in determining risk of future vascular complications [16] and is a time when vascular dysfunction is potentially reversible. Optimisation of diabetes control should be the initial strategy to improve vascular health in children and adolescents with T1D. However, glycosylated haemoglobin (HbA1c) levels are higher than target levels recommended for prevention of complications $[17,18]$. This is despite the advances in insulin delivery with insulin analogues and continuous subcutaneous insulin infusion (CSII) [19], highlighting the need to identify additional vascular protective strategies to prevent cardiovascular disease at its inception. The aim of this study is to determine whether metformin improves vascular health in children with T1D.

\section{Assessment of vascular health Vascular function}

The endothelium is a key regulator of vascular function [20] and endothelial dysfunction occurs early in the development of atherosclerosis [21]. Vascular dysfunction can be measured by Flow Mediated Dilatation (FMD) and Glyceryl Trinitrate mediated Dilatation (GTN). These are early, non-invasive markers of atherosclerosis and predate the development of clinically evident disease [22,23].

Ultrasound assesses brachial artery responses to increased blood flow (FMD). This induces nitric oxide release from the endothelium with a resultant increase in brachial artery diameter and is a measure of endothelial function. Exogenous administration of glyceryl trinitrate increases vessel diameter independent of the endothelium and is a measure of smooth muscle function (GTN). FMD and GTN have proven to be accurate and reproducible methods for assessment of vascular function [24].

Endothelial dysfunction of the coronary arteries, as measured by coronary artery vasomotor responses, is associated with a higher incidence of cardiovascular events in adulthood. FMD of the brachial artery relates to both coronary artery vasomotor responses and to the extent of coronary artery disease on coronary angiography findings
[25]. Reduced FMD in adults relates to traditional cardiovascular risk factors [22]. FMD is abnormal in children and young adults at risk of atherosclerosis [23]. FMD and GTN will be outcome measures in this study.

\section{Vascular structure}

The thickness of the intima and media layer of the carotid (cIMT) and aortic (aIMT) walls are structural markers of atherosclerosis and can be evaluated by ultrasound.

cIMT is a well-established index of early atherosclerosis that correlates with prevalent and incident coronary heart disease and stroke [26]. Relative risk of stroke or myocardial infarction in older adults without a history of cardiovascular disease increases by $35 \%$ with an increase in cIMT of $1 \mathrm{SD}$, i.e. a relatively large clinical effect for a small change in cIMT [26]. Overweight and obese children have increased cIMT compared with age matched controls [27]. cIMT has been used as a primary outcome measure in landmark intervention trials $[7,28]$. Diet and exercise improve endothelial dysfunction and reduce cIMT in obese children over 12 months [29]. In this study FMD improved early and was maintained and cIMT improved at 12 months. Atherosclerosis begins in the abdominal aorta. We and others have shown that aIMT precedes changes in cIMT in children with accelerated atherosclerosis, including T1D $[3,4]$. cIMT and aIMT will be used in this study as structural outcome measures.

\section{Adiponectin}

Adiponectin is an adipocytokine that regulates nitrous oxide by activating endothelial nitric oxide synthase. It may provide a measurable link between visceral obesity, insulin resistance and vascular dysfunction. We have shown that adiponectin levels relate to vascular smooth muscle function in obese youth [30]. Adiponectin/leptin ratio is an emerging measure of insulin resistance and is substantially higher in children with type 2 compared with type 1 diabetes [31,32].

\section{Metformin as a therapy to improve vascular health Mechanism of action}

Metformin is a biguanide that reduces glucose output from the liver and increases insulin sensitivity. Metformin activates the energy regulating AMP activated protein kinase (AMPK), principally in muscle and liver. This is the major mechanism of metformin's action, to increase insulin stimulated glucose uptake in skeletal muscle and adipocytes, and reduce hepatic glucose output. Metformin also activates AMPK in the endothelium and smooth muscle. This is likely to explain the improvement in endothelium dependent and independent vascular responses with the administration of metformin in adults with T1D and polycystic ovarian syndrome [33-35]. It may also explain the recognised benefits of 
metformin on cardiovascular risk independent of its glucose lowering effect. Metformin also improves adiponectin and leptin levels [35-37].

\section{Type 2 diabetes}

Metformin is the first line medication (with diet and exercise) in youth with type 2 diabetes (T2D) and/or with metabolic syndrome [38]. In T2D it improves endothelial function, decreases weight gain, triglycerides, LDL cholesterol and pro-inflammatory and pro-coagulation factors $[39,40]$. Importantly, in two large randomised trials metformin reduced the rate of myocardial infarction in adults with T2D by 33\%. Metformin also improves BMI, body composition and fasting insulin in obese youth without diabetes $[41,42]$.

\section{Type 1 diabetes}

A 6 month pilot study reported improvements in vascular health, as measured by FMD, in 44 adults with T1D treated with metformin or placebo in addition to insulin [33]. Meta-analysis of randomised studies of metformin in T1D shows benefits in reducing daily insulin requirement and reduced weight gain [43]. The potential benefit on improving LDL cholesterol is not confirmed and reductions in $\mathrm{HbA} 1 \mathrm{c}$ are inconsistent.

There are only two studies in children using metformin in addition to insulin. They show that metformin lowered HbA1c and decreased insulin dose without weight gain in children with poor metabolic control $[44,45]$.

Therefore, metformin is a logical adjunct treatment for T1D in addition to insulin. There are no data on the effects of metformin on vascular health in children and adolescents with T1D. We have shown that children with T1D have severe vascular dysfunction which relates to BMI [14]. We will therefore determine the effect of metformin on vascular function and structure in children with T1D and above average BMI, in the absence of any published trials.

\section{Aims and hypotheses}

The primary objective of this double blind, randomised, placebo controlled trial is to determine whether metformin improves vascular function as measured by FMD in children aged 8-18 years who have T1D.

The secondary objectives are to determine the effect of metformin on vascular health as measured by GTN, cIMT and aIMT in addition to other variables including adiponectin/leptin ratio, waist circumference, BMI, body composition, insulin requirements, HbA1c and lipids.

We hypothesise that the intervention group (those that receive metformin) will have a significant improvement in vascular health compared to the placebo group. We also hypothesise that those in the intervention group will have improvements in adiponectin/leptin ratio, waist circumference, BMI, body composition, insulin requirements, HbA1c and lipids.

\section{Methods/design}

\section{Approval and registration}

This study is a parallel, double blind, randomised placebo controlled trial over 12 months in two paediatric diabetes centres in Adelaide, South Australia. The trial has been approved by the Women's and Children's Hospital Research Ethics Committee (HREC 2327/12/13) and Flinders Medical Centre Research Ethics Committee (HREC 443.12) and is prospectively registered with the Australian New Zealand Clinical Trials Registry (ACTRN12611000148976).

All children with T1D who are seen in the diabetes outpatient clinics of the Women's and Children's Hospital (WCH) and Flinders Medical Centre (FMC) paediatrics department will be approached consecutively to be screened for eligibility in the study.

A total sample of 76 children aged $8-18$ years with T1D will be recruited. The treatment period is 12 months in duration. Written informed consent will be obtained from all parents of participants and written assent will be obtained from all participants.

\section{Inclusion criteria}

Each child must meet the following criteria to be involved in this study:

- diagnosed with type 1 diabetes

- aged between 8 and 18 years.

- $\mathrm{BMI}>50$ th centile for age and sex [Centers for Disease Control and Prevention 2000 standardized reference charts (http://wwwn.cdc.gov/epiinfo/)].

- T1D duration greater than 1 year

- insulin requirements $>0.5$ units $/ \mathrm{kg} /$ day to exclude subjects in the remission phase of T1D

\section{Exclusion criteria}

Children are excluded from the study if they meet any of the following criteria:

- non T1D i.e. T2D or other forms of diabetes

- severe hypoglycaemic episode in preceding 6 months defined as a loss of consciousness or convulsion associated with hypoglycaemia

- recurrent diabetic ketoacidosis (more than 2 episodes in the preceding year)

- other serious co-morbidities but not including treated hypothyroidism or coeliac disease

- contraindications to metformin therapy: hypersensitivity to metformin, renal or liver dysfunction, vitamin B12 deficiency, inability to abstain from alcohol

- pregnancy or breast feeding 
- subjects taking metformin, statins, multivitamins, or anti-hypertensives

\section{Randomisation}

The study has a planned sample size of 76 with 38 in each group. Randomisation was performed by an independent statistician using statistical software S-plus version 8.1 to provide equal representation of placebo and metformin. Allocation concealment was used to implement the random sequence allocation.

Participants will be recruited from paediatric outpatient clinics at WCH and FMC by a single investigator (JA). Participants will be assigned randomisation numbers by the investigator in sequence and allocated to a treatment group by the pharmacist using the generated randomisation list. Treatment allocation will be concealed from all study investigators, the distributing pharmacist and participants to minimise potential bias.

Analysis of the data will be performed by a statistician (TS) who was not involved in the initial randomisation.

\section{Participant withdrawal}

Any participant may terminate participation in the study without giving a reason and without any disadvantage or interference with the diabetes care provided to the participant at the treating hospital. An investigator can stop the participation of a subject after consideration of the benefit/risk ratio of participation in the trial. Reasons to stop participation include: serious adverse events, request by safety committee of early termination due to safety reasons and limitation to continue the trial such as a participant moving interstate.

\section{Treatment arms and dosage of medication}

The children will receive either metformin or placebo according to their randomly allocated group. The study procedure details are outlined in Figure 1. Metformin $500 \mathrm{mg}$ oral tablets will be supplied by GenericHealth (Camberwell, Victoria, Australia). The placebo will be also supplied by the same company and is identical in appearance and ingredients, aside from the active ingredient, metformin hydrochloride. If the participant's weight is less than $60 \mathrm{~kg}$ they will be assigned to a dosage of $500 \mathrm{mg}$ twice a day (titrated up over 2 weeks). If the participant weighs greater than $60 \mathrm{~kg}$ they will be assigned to a dosage of $1 \mathrm{~g}$ twice a day (titrated up over 6 weeks). Dose titration will be limited to tolerance of the medication. If a participant experiences a reduction in insulin dose of greater than $20 \%$, or if they have persistent gastrointestinal side effects, they will be prescribed the maximum tolerated dose.

Both groups will also receive a dietary intervention at the commencement of the study and at 3 months. At the first visit, all subjects will receive standardised information regarding healthy diet, portion sizes, regular meals and physical activity. This information will be delivered using a 'Type 1 diabetes and healthy weight' resource Additional file 1), developed by our dietician (BD) using current guidelines for healthy eating in T1D.

At the 3 month assessment, all participants will take part in a standardised interactive nutrition session developed by

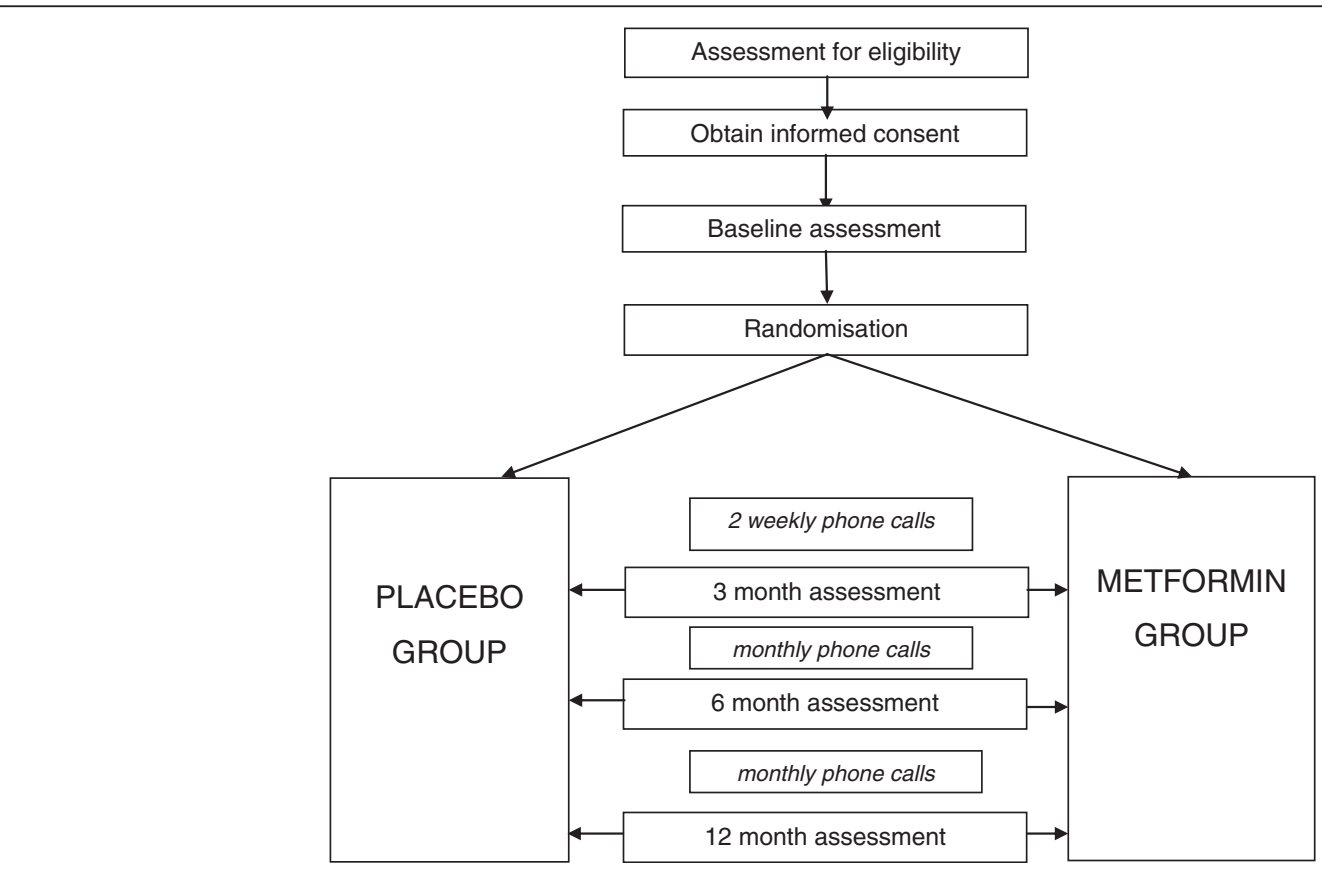

Figure 1 Study procedure details. 
BD comparing the relative nutritional value of healthy snack foods to 'sometimes' and 'treat' snack foods.

\section{Outcome measures}

Table 1 shows which primary and secondary outcomes will be measured at different time points in the study. Table 2 shows additional information which will be collected at the assessments and Table 3 shows the additional information collected at each telephone call (which occurs 2 weekly for the first 3 months and monthly thereafter).

\section{Ultrasound assessment of vascular function (FMD and GTN) and vascular structure (cIMT and aIMT)}

Experienced and blinded sonographers (trained and led by RG) will perform all B mode ultrasound examinations with a $17-5 \mathrm{MHz}$ linear array transducer (iU22; Phillips,
Bothel, Washington, USA) in a temperature-controlled room $\left(22-24^{\circ} \mathrm{C}\right)$.

\section{Flow mediated dilatation (FMD) and glyceryl trinitrate mediated dilatation (GTN)}

FMD and GTN will be assessed as previously described $[3,14,46,47]$. Ultrasound images of the brachial artery in longitudinal section, $2-15 \mathrm{~cm}$ above the elbow will allow measurements of the arterial diameter. Each study will include 4 scans: (1) resting scan; subsequently, reactive hyperaemia is induced by occluding arterial blood flow using a sphygmomanometer inflated to $250 \mathrm{mmHg}$ for 4 minutes; (2) FMD scan recorded between 45-75 seconds after cuff deflation; (3) re-control scan 10-15 minutes later (allowing for vessel recovery); and (4) last scan, taken 4 minutes after the sublingual administration of the GTN spray (400 $\mu \mathrm{g}$, Nitrolingual Pump spray,

Table 1 Primary and secondary outcome measures

\begin{tabular}{|c|c|c|c|c|}
\hline & Baseline & 3 months & 6 months & 12 months \\
\hline \multicolumn{5}{|l|}{ Primary outcome } \\
\hline Vascular endothelial function (FMD) & * & * & * & * \\
\hline \multicolumn{5}{|l|}{ Secondary outcomes } \\
\hline Vascular smooth muscle function (GTN) & * & * & * & * \\
\hline Vascular structure (cIMT and alMT) & * & & & * \\
\hline Physical examination & * & * & * & * \\
\hline \multicolumn{5}{|l|}{ - anthropometric measurements (height, weight, BMI, waist circumference) } \\
\hline \multicolumn{5}{|l|}{ - the presence of acanthosis nigricans } \\
\hline \multicolumn{5}{|l|}{ - severity of acne } \\
\hline \multicolumn{5}{|l|}{ - ferriman - gallwey score (girls) } \\
\hline Frequency and severity of hypoglycaemia & * & * & * & * \\
\hline Insulin dose (units/kg/day) & * & * & * & * \\
\hline Glycosylated haemoglobin & * & * & * & * \\
\hline Fasting glucose & * & * & * & * \\
\hline Fasting total/HDL/LDL cholesterol/triglycerides & * & * & * & * \\
\hline High sensitivity C-Reactive Protein (HsCRP) & * & * & * & * \\
\hline Early morning urinary albumin/creatinine & * & * & * & * \\
\hline Urinary prostaglandin $\mathrm{F} 2 \mathrm{a}$ & * & * & * & * \\
\hline Liver function, renal function tests and lactate & * & * & * & * \\
\hline Total Plasma homocysteine & * & * & * & * \\
\hline $\begin{array}{l}\text { Girls only: Testosterone, free androgen index, Sex Hormone-Binding } \\
\text { Globulin, Dehydroepiandrosterone, Anti-Mullerian Hormone, 17-hydroxy } \\
\text { progesterone, prolactin, Luteinizing hormone, Follicle Stimulating Hormone, } \\
\text { progesterone \& oestradiol }\end{array}$ & * & * & * & * \\
\hline Total Adiponectin & * & * & * & * \\
\hline Leptin & * & * & * & * \\
\hline $\begin{array}{l}\text { Body composition (bio-electrical impedance analysis } \\
\text { using Tanita body composition scales) }\end{array}$ & * & * & * & * \\
\hline Retinal photograph & * & & & * \\
\hline Total body Dual-energy X-ray absorptiometry scan & * & & & * \\
\hline
\end{tabular}

*Indicates assessment of a variable at the timepoint indicated. 
Table 2 Additional information collected at assessments

\begin{tabular}{|c|c|c|c|c|}
\hline & Baseline & 3 months & 6 months & 12 months \\
\hline ACAES questionnaire $v 1.2$ & * & * & * & * \\
\hline Physical examination - tanner stage & * & * & * & * \\
\hline Daily energy expenditure (SenseWear arm band) & * & * & * & * \\
\hline Family history of premature cardiovascular disease & * & & & * \\
\hline Pregnancy test in post menarchal girls & * & * & * & * \\
\hline Serum cotinine & * & * & * & * \\
\hline Adherence data using MEMS caps and manual tablet count & * & * & * & * \\
\hline Serum Vitamin B12, Folate, red cell folate & * & * & * & * \\
\hline
\end{tabular}

* Indicates assessment of a variable at the timepoint indicated.

manufactured by G. Pohl-Boskamp GmbH \& co. KG Hohenlockstedt, Germany, distributed by Sanofi-Aventis, Macquarie Park, NSW, Australia). For each scan, measurements will be made over 4 consecutive cardiac cycles, incident with the R wave on the ECG (i.e., at the end of diastole), by observers blinded to the intervention type, using ultrasonic calipers. Measurements will be averaged and expressed as percentages of the resting scan. Our coefficient of variation $(\mathrm{CV})$ between 20 controls is $3.9 \%$ for FMD and 4.0\% for GTN-mediated dilatation [46].

\section{Carotid and aortic intima media thickness (cIMT and alMT)}

cIMT and aIMT will be assessed as previously described $[3,48]$. In brief, the left and right common carotid artery will be imaged in a standardized magnification $(2 \times$ $2 \mathrm{~cm}$ ) using images of the posterior (far) wall of the distal $10 \mathrm{~mm}$ of the common carotid artery, just proximal to the carotid bulb. Optimal images will be captured at the end of diastole, incident with the R-wave of the ECG, for later analysis. The greatest distance between the lumen-intima interface and media -adventitia interface (IMT) is measured using an automatic edge detection and measurement computer software package, and mean and maximum IMT are recorded. The mean of 2 measurements for aIMT and 6 measurements ( 3 on each side) for cIMT will be calculated according to the current Gold Standard protocol for cIMT. Analysis will be by two blinded observers. Sonographers have been trained and have undergone an accreditation process to

Table 3 Additional information collected with each phone call

\begin{tabular}{lcc}
\hline & $\mathbf{0 - 3}$ months & $\mathbf{3 - 1 2}$ months \\
\hline Side effects & $\begin{array}{c}2 \text { weekly } \\
\text { telephone calls }\end{array}$ & $\begin{array}{c}\text { Monthly } \\
\text { telephone calls }\end{array}$ \\
- hypoglycaemia (number, severity) & \\
- gastrointestinal symptoms & \\
(nausea, vomiting, diarrhoea, anorexia) & \\
- rash & \\
- other side effects & \\
Insulin requirements & \\
\hline
\end{tabular}

evaluate the quality of their scans for this study in 2009 . Our inter-observer intra-class coefficient for cIMT measurements is $0.99(\mathrm{CV} 1.2 \%)$ and the intra-observer intra-class coefficient for cIMT measurements is 0.97 (CV 2.4\%) [3].

\section{Physical examination}

Weight and body composition will be measured in light clothing using BC-418 segmental body composition analyser (Tanita, Tokyo, Japan distributed by Wedderburn, Inglewood, NSW, Australia). Height will be measured on a wall mounted stadiometer (to $0.1 \mathrm{~cm}$ ). Waist and hip circumference will be measured with a tape (to $0.5 \mathrm{~cm}$ ). Waist circumference will be measured at the midpoint between the lower edge of the ribs in the mid-axillary line and the top of the iliac crest, at minimal respiration. Hip circumference will be measured at the maximum circumference of the buttocks with the tape parallel to the floor. BMI z score will be calculated using 3.2 EpiInfo database version. 2 and Centers for Disease Control and Prevention 2000 standardized reference charts (http:// wwwn.cdc.gov/epiinfo/). Blood pressure will be measured using DINAMAP (Carescape V100 Vital signs monitor, GE Healthcare, Milwauke, WI) with appropriate-size cuff on the left arm after 10 minutes of rest in supine position. The mean of 3 consecutive measurements will be recorded.

\section{Laboratory methods}

Cholesterol (LDL and HDL) and triglycerides will be measured using commercial enzymatic assays on Roche/ Hitachi cobas $\mathrm{C}$ systems. High sensitivity $\mathrm{C}$ reactive protein (hs CRP) will be measured using a near infrared particle immunoassay method using IMMAGE Immunochemistry Systems Reagent (Beckman Coulter Inc, Fullerton, California, USA). Glycosylated haemoglobin (HbA1c) will be measured using a latex immunoagglutination inhibition methodology (DCA 2000 Hemoglobin A1c Reagent Kit; Bayer, Toronto, Ontario). Serum cotinine will be measured using a cotinine micro-plate EIA (STC Technologies). Urinary albumin/creatinine will be 
measured by immunoturbidometric and enzymatic colorimetric methods in routine laboratory assays. Oxidative stress will be assessed by measuring urinary PGF $2 \alpha$ using a competitive enzyme-linked immunoassay and values obtained will be corrected for urinary creatinine. Liver function and renal function tests will be measured by Roche/Hitachi cobas $\mathrm{C}$ systems.

Serum samples will be frozen at -80 degrees Celsius for later measurement of adiponectin, leptin and androgens. Total adiponectin and leptin will be analysed using an enzyme-linked immunoassay (ALPCO diagnostics, Salem, NH, USA). Serum androgens will be measured by liquid chromatography mass spectroscopy (CPR Pharma Services, Adelaide, South Australia).

\section{Total body dual-energy X-ray absorptiometry scan}

The ratio of total body fat (g) to lean body mass (g) will be measured using dual-energy X-ray absorptiometry (DXA) on a GE-Lunar Prodigy machine (GE LunarCorp, Maddison, WI) equipped with adult property software, version 3.6, which is appropriate for body weight of subjects 8-18 years. Fast scan mode and standard subject positioning will be used for these measurements. Mean precision of our machine is $1.3 \%$ for soft tissue.

\section{Assessment of energy intake and diet quality}

The Australian Child and Adolescent Eating Survey (ACAES - version 1.2) [49] is a food frequency questionnaire based on the previous 3-6 months of food intake across all food categories. It will be intervieweradministered by the same dietitian at each assessment. At baseline, the ACAES will calculate the dietary intake over the previous 6 months. At subsequent assessments (3, 6 and 12 months) the ACAES questionnaire will calculate the dietary intake since the previous assessment. The ACAES will determine total caloric intake and macro and micronutrient content of the participant's diet.

\section{Assessment of energy expenditure}

Daily energy expenditure will be measured at 0, 3, 6 and 12 months. This will be assessed with the use of a SenseWear MF (Mini Form factor) device (analysed with SenseWear Software Version 7.0. Temple Healthcare Pty Ltd, Bowral, Australia) which is an external armband worn in this study for 5 days before each assessment time point. Based on measurements of accelerometry, heat flux, galvanic skin response, skin temperature, near-body temperature and patient characteristics (gender, age, height, weight) proprietary algorithms will calculate energy expenditure, number of steps taken and sleep duration.

\section{Monitoring of safety and adverse events Blood glucose management during study}

All subjects will receive 2 weekly telephone review of blood glucose control for the first 3 months and monthly thereafter. They will also have access to a 24 hour phone hotline serviced by one of 4 paediatric endocrinologists for acute insulin dose adjustment.

\section{Compliance assessment}

Assessment of adherence to the study medication regimen will be assessed at each assessment time point (3 months, 6 months and 12 months). All unused study drugs will be returned to the investigator for counting. MEMS caps (AARDEX group LTD, Sion, Switzerland) will also be utilised to measure compliance. These caps record how many times the study medication bottle is opened between study visits and the data will be downloaded for review and analysis. Compliance will be encouraged at the 3 month and 6 month study visit using the downloaded data from the MEMS cap.

\section{Monitoring of adverse events}

Renal, liver function tests and lactate will be performed at 0, 3, 6 and 12 months. Metformin can decrease levels of vitamin B12, so children with vitamin B12 deficiency will be excluded and vitamin B12 will be monitored during the study. The risk of hypoglycaemia will be monitored and insulin will be adjusted accordingly.

Metformin can cause nausea and diarrhoea. Any illness or need for additional medication in a subject will be recorded throughout the study. Letters to the subjects' endocrinologists and general practitioner advising of entry into the trial will be sent, and the $\mathrm{WCH}$ and FMC emergency departments will have a record of the subject's participation in the trial in the medical records.

All adverse events will be reported to the medication safety committee for the trial in a monthly monitoring update. All serious adverse events will be reported to the medication safety committee and the $\mathrm{WCH}$ human research ethics committee within 24 hours of the event occurring. Serious adverse events occuring in FMC participants will also be reported to the FMC human research ethics committee. A serious adverse event is defined as one which is fatal or life threatening or requires hospitalization.

\section{Data analysis}

All analyses will be performed under the intention to treat principle. The primary outcome, FMD, will be compared between metformin and placebo groups over 12 months using a linear mixed effects model. Linear mixed effects models will also be used to compare changes in secondary continuous outcomes (GTN, adiponectin/leptin ratio, lipids, HbA1c, insulin requirements, cIMT and aIMT) 
between treatment groups (metformin/placebo) over the four reviews. The linear mixed effects models will account for the within-subject correlations that are produced when repeating outcome measurements in individual subjects over time. Both unadjusted and adjusted analyses will be performed, with adjustment for important pre-specified baseline covariates including age, sex and BMI.

While no studies have looked at the effect of metformin on FMD in T1D in this age group, our previous work found an improvement in FMD of 3.1\% with an SD of 4.3 in children with T1D receiving folic acid over 8 weeks $[46,47]$. Assuming an equivalent improvement with metformin from baseline to 12 months, we would require 32 subjects per group to have $80 \%$ power to detect a difference in FMD changes of 3.1\% between randomised groups (alpha $=0.05$ two-sided). Our dropout rate in previous studies in this population with similar intervention and investigation over 24 weeks has been $1-2 \%$. We have conservatively factored in a dropout rate of $15 \%$ giving a total recruitment required of 76 subjects (38 in each group).

\section{Discussion}

Cardiovascular disease is the commonest cause of morbidity and mortality in T1D. The origins of cardiovascular disease are in childhood. Good metabolic control is essential in the prevention of cardiovascular disease however targets for optimal glycaemic control are often not met in children and adolescents with T1D. It is crucial to investigate additional effective and early interventions to prevent or reduce vascular pathology in this population. Metformin is a good candidate for early prevention of cardiovascular disease as it has been prescribed in youth with T2D for over 10 years and reduces cardiovascular disease in T2D.

Metformin is generally well tolerated and easy to administer with a good safety profile. Vascular health outcomes will be measured using non invasive, valid and reliable measurements by experienced investigators. This is, to our knowledge, the first study to examine whether administration of metformin will improve vascular health in children with T1D. Determining the effect of metformin on vascular health in children with T1D will provide a potential early intervention for cardiovascular disease in this population.

\section{Additional file}

Additional file 1: "Type 1 Diabetes and healthy weight" dietician resource used for dietary intervention at baseline assessment.

\section{Abbreviations}

ACAES: Australian child and adolescent eating survey; alMT: Aortic intima media thickness; AMPK: AMP activated protein kinase; BMI: Body mass index; CIMT: Carotid intima media thickness; CSII: Continuous subcutaneous insulin infusion; DXA: Dual-energy X-ray absorpitometry; FMC: Flinders Medical Centre; FMD: Flow mediated dilatation; GTN: GTN mediated dilatation; HbA1c: Glycosylated haemoglobin; IMT: Intima media thickness; T1D: Type 1 diabetes; T2D: Type 2 diabetes; WCH: Women's and Children's Hospital.
Competing interests

The authors declare that they have no competing interests.

\section{Authors' contributions}

$J C$ and AP received study funding. AP, JC and JA conceived of the study. All authors participated in study design. TS performed sample size calculation, planned the statistical analysis and will be involved in study analysis. RG, lead sonographer, reviewed the vascular health assessment methods and will supervise scan quality. BD designed the dietary intervention and created the resource "Type 1 Diabetes and Healthy Weight". TO assisted in evaluation of physical activity and energy expenditure for the study. BC assisted with the ethics application for FMC and will supervise the FMC patients. JA, AP and JC drafted the manuscript. All authors read and corrected draft versions and approved the final manuscript.

\section{Acknowledgements}

This study is funded by Diabetes Australia Research Trust (DART), Women's and Children's Research Foundation and Australasian Paediatric Endocrine Care (APEC) grants. Our thanks to Kate Dowling, statistician, who performed the randomisation for this study.

\section{Author details}

${ }^{1}$ School of Paediatrics and Reproductive Health, University of Adelaide, Adelaide, Australia. 'Department of Paediatric Endocrinology, Women's and Children's Hospital, Adelaide, Australia. ${ }^{3}$ Department of Public Health, University of Adelaide, Adelaide, Australia. ${ }^{4}$ Department of Medical Imaging, Women's and Children's Hospital, Adelaide, Australia. ${ }^{5}$ Department of Nutrition and Dietetics, Women's and Children's Hospital, Adelaide, Australia. ${ }^{6}$ School of Health Sciences, University of South Australia, Adelaide, Australia.

${ }^{7}$ Department of Paediatrics, Flinders Medical Centre, Adelaide, Australia.

Received: 3 July 2013 Accepted: 11 July 2013

Published: 16 July 2013

\section{References}

1. Orchard TJ, Costacou T, Kretowski A, Nesto RW: Type 1 diabetes and coronary artery disease. Diabetes Care 2006, 29(11):2528-2538.

2. Soedamah-Muthu SS, Fuller JH, Mulnier HE, Raleigh VS, Lawrenson RA, Colhoun HM: High risk of cardiovascular disease in patients with type 1 diabetes in the U.K: a cohort study using the general practice research database. Diabetes Care 2006, 4:798-804.

3. Harrington J, Pena AS, Gent R, Hirte C, Couper J: Aortic intima media thickness is an early marker of atherosclerosis in children with type 1 diabetes mellitus. J Pediatr 2010, 156(2):237-241.

4. Jarvisalo MJ, Jartti L, Nanto-Salonen K, Irjala K, Ronnemaa T, Hartiala JJ, Celermajer DS, Raitakari OT: Increased aortic intima-media thickness: a marker of preclinical atherosclerosis in high-risk children. Circulation 2001, 104(24):2943-2947.

5. Pena AS, Wiltshire E, MacKenzie K, Gent R, Piotto L, Hirte C, Couper J: Vascular endothelial and smooth muscle function relates to body mass index and glucose in obese and nonobese children. $J$ Clin Endocrinol Metab 2006, 91(11):4467-4471.

6. Elhadd TA, Kennedy G, Hill A, McLaren M, Newton RW, Greene SA, Belch JJ: Abnormal markers of endothelial cell activation and oxidative stress in children, adolescents and young adults with type 1 diabetes with no clinical vascular disease. Diabetes Metab Res Rev 1999, 15(6):405-411.

7. White NH, Cleary PA, Dahms W, Goldstein D, Malone J, Tamborlane WV: Beneficial effects of intensive therapy of diabetes during adolescence: outcomes after the conclusion of the Diabetes Control and Complications Trial (DCCT). J Pediatr 2001, 139(6):804-812.

8. Knerr I, Wolf J, Reinehr T, Stachow R, Grabert M, Schober E, Rascher W, Holl RW: The 'accelerator hypothesis': relationship between weight, height, body mass index and age at diagnosis in a large cohort of 9,248 German and Austrian children with type 1 diabetes mellitus. Diabetologia 2005, 48(12):2501-2504.

9. Gimenez M, Aguilera E, Castell C, De Lara N, Nicolau J, Conget I: Relationship between $\mathrm{BMI}$ and age at diagnosis of type 1 diabetes in a Mediterranean area in the period of 1990-2004. Diabetes Care 2007, 30(6):1593-1595. 
10. Conway B, Miller RG, Costacou T, Fried L, Kelsey S, Evans RW, Orchard TJ: Temporal patterns in overweight and obesity in Type 1 diabetes. Diabetic medicine: a journal of the British Diabetic Association 2010, 27(4):398-404

11. Purnell JQ, Zinman B, Brunzell JD: The effect of excess weight gain with intensive diabetes mellitus treatment on cardiovascular disease risk factors and atherosclerosis in type 1 diabetes mellitus: results from the Diabetes Control and Complications Trial/Epidemiology of Diabetes Interventions and Complications Study (DCCT/EDIC) study. Circulation 2013, 127(2):180-187.

12. Van Vliet M, Van der Heyden JC, Diamant M, Van der Rosenstiel IA, Schindhelm RK, Aanstoot HJ, Veeze HJ: Overweight is highly prevalent in children with type 1 diabetes and associates with cardiometabolic risk. J Pediatr 2010, 156(6):923-929.

13. Liu LL, Lawrence JM, Davis C, Liese AD, Pettitt DJ, Pihoker C, Dabelea D, Hamman R, Waitzfelder B, Kahn HS: Prevalence of overweight and obesity in youth with diabetes in USA: the SEARCH for Diabetes in Youth study. Pediatr Diabetes 2010, 11(1):4-11.

14. MacKenzie KE, Wiltshire EJ, Gent R, Hirte C, Piotto L, Couper JJ: Folate and vitamin $\mathrm{B} 6$ rapidly normalize endothelial dysfunction in children with type 1 diabetes mellitus. Pediatrics 2006, 118(1):242-253.

15. Szadkowska A, Pietrzak I, Szlawska J, Kozera A, Gadzicka A, Mlynarski W: Abdominal obesity, metabolic syndrome in type 1 diabetic children and adolescents. Pediatr Endocrinol Diabetes Metab 2009, 15(4):233-239.

16. Davis PH, Dawson JD, Riley WA, Lauer RM: Carotid intimal-medial thickness is related to cardiovascular risk factors measured from childhood through middle age: the muscatine study. Circulation 2001, 104(23):2815-2819.

17. Hanberger L, Samuelsson U, Lindblad B, Ludvigsson J: A1C in children and adolescents with diabetes in relation to certain clinical parameters: the Swedish Childhood Diabetes Registry SWEDIABKIDS. Diabetes Care 2008, 31(5):927-929.

18. Craig ME, Handelsman P, Donaghue KC, Chan A, Blades B, Laina R, Bradford $D$, Middlehurst A, Ambler G, Verge CF, et al: Predictors of glycaemic control and hypoglycaemia in children and adolescents with type 1 diabetes from NSW and the ACT. The Medical journal of Australia 2002, 177(5):235-238

19. Pankowska E, Blazik M, Dziechciarz P, Szypowska A, Szajewska H: Continuous subcutaneous insulin infusion vs. multiple daily injections in children with type 1 diabetes: a systematic review and meta-analysis of randomized control trials. Pediatr Diabetes 2009 10(1):52-58

20. Furchgott RF, Zawadzki JV: The obligatory role of endothelial cells in the relaxation of arterial smooth muscle by acetylcholine. Nature 1980, 288(5789):373-376.

21. Ross R: The pathogenesis of atherosclerosis: a perspective for the $1990 \mathrm{~s}$. Nature 1993, 362(6423):801-809.

22. Benjamin EJ, Larson MG, Keyes MJ, Mitchell GF, Vasan RS, Keaney JF Jr, Lehman BT, Fan S, Osypiuk E, Vita JA: Clinical correlates and heritability of flow-mediated dilation in the community: the Framingham Heart Study. Circulation 2004, 109(5):613-619.

23. Celermajer DS, Sorensen KE, Gooch VM, Spiegelhalter DJ, Miller OI, Sullivan ID, Lloyd JK, Deanfield JE: Non-invasive detection of endothelial dysfunction in children and adults at risk of atherosclerosis. Lancet 1992, 340(8828):1111-1115.

24. Sorensen KE, Celermajer DS, Spiegelhalter DJ, Georgakopoulos D, Robinson J, Thomas O, Deanfield JE: Non-invasive measurement of human endothelium dependent arterial responses: accuracy and reproducibility. Br Heart J 1995, 74(3):247-253.

25. Neunteufl T, Katzenschlager R, Hassan A, Klaar U, Schwarzacher S, Glogar D, Bauer $\mathrm{P}$, Weidinger F: Systemic endothelial dysfunction is related to the extent and severity of coronary artery disease. Atherosclerosis 1997, 129(1):111-118.

26. Bots ML, Grobbee DE, Hofman A, Witteman JC: Common carotid intima-media thickness and risk of acute myocardial infarction: the role of lumen diameter. Stroke 2005, 36(4):762-767.

27. Woo KS, Chook P, Yu CW, Sung RY, Qiao M, Leung SS, Lam CW, Metrewel C, Celermajer DS: Overweight in children is associated with arterial endothelial dysfunction and intima-media thickening. Int J Obes Relat Metab Disord 2004, 28(7):852-857.
28. Chahal H, Backlund JY, Cleary PA, Lachin JM, Polak JF, Lima JA, Bluemke DA: Relation between carotid intima-media thickness and left ventricular mass in type 1 diabetes mellitus (from the Epidemiology of Diabetes Interventions and Complications [EDIC] Study). Am J Cardiol 2012, 110(10):1534-1540

29. Woo KS, Chook P, Yu CW, Sung RY, Oiao M, Leung SS, Lam CW, Metreweli C, Celermajer DS: Effects of diet and exercise on obesity-related vascular dysfunction in children. Circulation 2004, 109(16):1981-1986.

30. Pena AS, Belobrajdic DP, Wiltshire E, Gent R, Hirte C, Couper J: Adiponectin relates to smooth muscle function and folate in obese children. International journal of pediatric obesity: IJPO: an official journal of the International Association for the Study of Obesity 2010, 5(2):185-191.

31. Zaletel J, Barlovic DP, Prezelj J: Adiponectin-leptin ratio: a useful estimate of insulin resistance in patients with Type 2 diabetes. $J$ Endocrinol Invest 2010, 33(8):514-518

32. Maahs DM, Hamman RF, D'Agostino R Jr, Dolan LM, Imperatore G, Lawrence JM, Marcovina SM, Mayer-Davis EJ, Pihoker C, Dabelea D: The association between adiponectin/leptin ratio and diabetes type: the SEARCH for Diabetes in Youth Study. J Pediatr 2009, 155(1):133-135. 135 e131.

33. Pitocco D, Zaccardi F, Tarzia P, Milo M, Scavone G, Rizzo P, Pagliaccia F, Nerla R, Di Franco A, Manto A, et al: Metformin improves endothelial function in type 1 diabetic subjects: a pilot, placebo-controlled randomized study. Diabetes Obes Metab 2013, 15(5):427-431.

34. Davis BJ, Xie Z, Viollet B, Zou MH: Activation of the AMP-activated kinase by antidiabetes drug metformin stimulates nitric oxide synthesis in vivo by promoting the association of heat shock protein 90 and endothelial nitric oxide synthase. Diabetes 2006, 55(2):496-505.

35. Agarwal N, Rice SP, Bolusani H, Luzio SD, Dunseath G, Ludgate M, Rees DA Metformin reduces arterial stiffness and improves endothelial function in young women with polycystic ovary syndrome: a randomized, placebo-controlled, crossover trial. J Clin Endocrinol Metab 2010, 95(2):722-730

36. Morin-Papunen LC, Koivunen RM, Tomas C, Ruokonen A, Martikainen HK Decreased serum leptin concentrations during metformin therapy in obese women with polycystic ovary syndrome. J Clin Endocrinol Metab 1998, 83(7):2566-2568.

37. Zulian A, Cancello R, Girola A, Gilardini L, Alberti L, Croci M, Micheletto G, Danelli $P$, Invitti $C$ : In vitro and in vivo effects of metformin on human adipose tissue adiponectin. Obes Facts 2011, 4(1):27-33.

38. Zeitler P, Hirst K, Pyle L, Linder B, Copeland K, Arslanian S, Cuttler L, Nathan DM, Tollefsen S, Wilfley D, et al: A clinical trial to maintain glycemic control in youth with type 2 diabetes. N Eng J Med 2012, 366(24):2247-2256.

39. Mather KJ, Verma S, Anderson TJ: Improved endothelial function with metformin in type 2 diabetes mellitus. J Am Coll Cardiol 2001, 37(5):1344-1350.

40. Wulffele MG, Kooy A, De Zeeuw D, Stehouwer CD, Gansevoort RT: The effect of metformin on blood pressure, plasma cholesterol and triglycerides in type 2 diabetes mellitus: a systematic review. J Intern Med 2004, 256(1):1-14

41. Kendall D, Vail A, Amin R, Barrett $T$, Dimitri $P$, Ivison F, Kibirige M, Mathew $V$, Matyka K, McGovern A, et al: Metformin in obese children and adolescents: the MOCA trial. J Clin Endocrinol Metab 2013, 98(1):322-329.

42. Srinivasan S, Ambler GR, Baur LA, Garnett SP, Tepsa M, Yap F, Ward GM, Cowell CT: Randomized, controlled trial of metformin for obesity and insulin resistance in children and adolescents: improvement in body composition and fasting insulin. J Clin Endocrinol Metab 2006, 91(6):2074-2080.

43. Vella $S$, Buetow L, Royle P, Livingstone S, Colhoun HM, Petrie JR: The use of metformin in type 1 diabetes: a systematic review of efficacy. Diabetologia 2010, 53(5):809-820.

44. Hamilton J, Cummings E, Zdravkovic V, Finegood D, Daneman D: Metformin as an adjunct therapy in adolescents with type 1 diabetes and insulin resistance: a randomized controlled trial. Diabetes Care 2003, 26(1):138-143.

45. Sarnblad S, Kroon M, Aman J: Metformin as additional therapy in adolescents with poorly controlled type 1 diabetes: randomised placebo-controlled trial with aspects on insulin sensitivity. European journal of endocrinology / European Federation of Endocrine Societies 2003, 149(4):323-329. 
46. Wiltshire EJ, Gent R, Hirte C, Pena A, Thomas DW, Couper JJ: Endothelial dysfunction relates to folate status in children and adolescents with type 1 diabetes. Diabetes 2002, 51:2282-2286.

47. Pena AS, Wiltshire E, Gent R, Hirte C, Couper J: Folic acid improves endothelial function in children and adolescents with type 1 diabetes. J Pediatr 2004, 144(4):500-504.

48. Harrington J, Pena AS, Wilson L, Gent R, Dowling K, Baghurst P, Couper J: Vascular function and glucose variability improve transiently following initiation of continuous subcutaneous insulin infusion in children with type 1 diabetes. Pediatr Diabetes 2013: Epub ahead of print.

49. Watson JF, Collins CE, Sibbritt DW, Dibley MJ, Garg ML: Reproducibility and comparative validity of a food frequency questionnaire for Australian children and adolescents. Int J Behav Nutr Phys Act 2009, 6:62.

doi:10.1186/1471-2431-13-108

Cite this article as: Anderson et al: Does metformin improve vascular health in children with type 1 diabetes? Protocol for a one year, double blind, randomised, placebo controlled trial. BMC Pediatrics 2013 13:108.

\section{Submit your next manuscript to BioMed Central and take full advantage of:}

- Convenient online submission

- Thorough peer review

- No space constraints or color figure charges

- Immediate publication on acceptance

- Inclusion in PubMed, CAS, Scopus and Google Scholar

- Research which is freely available for redistribution 